352

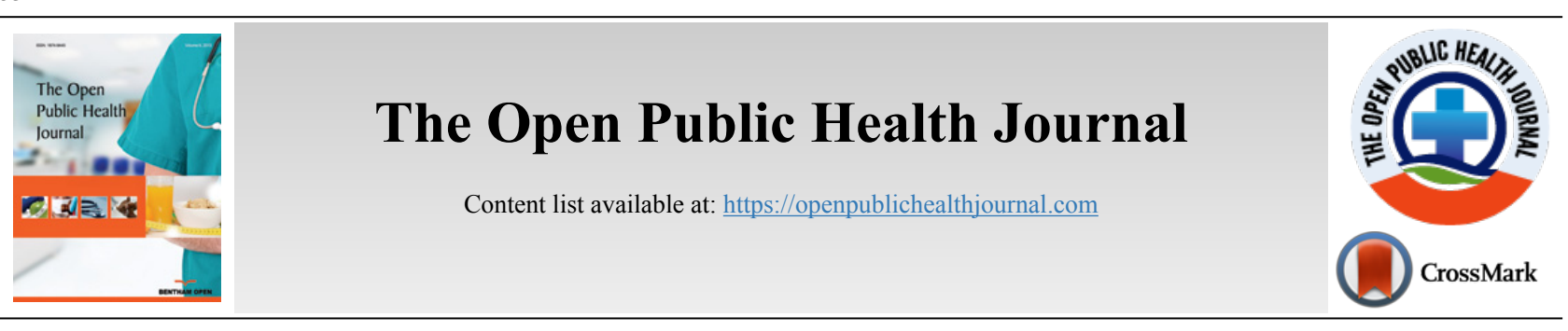

RESEARCH ARTICLE

\title{
COVID-19 Vaccine Hesitancy among Health Care Workers in the Middle East Region
}

\author{
Ahmed H. Aoun ${ }^{1,2,}$, Mohamed H. Aon ${ }^{3,4}$, Abdulrahman Z. Alshammari ${ }^{3}$ and Shady A Moussa ${ }^{5}$ \\ ${ }^{I}$ Department of Pediatrics, Faculty of Medicine, Cairo University, Giza, Egypt. \\ ${ }^{2}$ Primary Health Care Corporation, Doha, Qatar. \\ ${ }^{3}$ Department of Medicine, Jahra Hospital, Al-Jahra, Kuwait. \\ ${ }^{4}$ Department of Medicine, Faculty of Medicine, Cairo University, Giza, Egypt. \\ ${ }^{5}$ Department of Pediatric Dentistry and Dental Public Health, Zagazig University, Zagazig, Egypt.
}

\begin{abstract}
:
Background:

Coronavirus disease 2019 (COVID-19) vaccine development is the best approach to fight the disease. However, rising vaccine hesitancy can make widespread vaccine application difficult.

Objectives:

To explore health care workers' attitudes towards the COVID-19 vaccine and find the reasons lying behind vaccine hesitancy among participants.

Methods:

Our study was a cross-sectional survey. An anonymous online questionnaire was sent to a sample of health care workers living and working in the Middle East region. Data collected included demographic data, educational attainment, household crowding, risk factors for severe COVID-19
\end{abstract} infection, influenza vaccination history, and questions about COVID-19 vaccine acceptance.

Results:

We received 864 validated responses. The study included 365 physicians, 391 nurses, and 108 allied professions. Females represented $61 \%$ of participants and $98.5 \%$ of participants were below 65 years old. Around $60 \%$ of participants were hesitant to get the COVID-19 vaccine. The commonest reasons for hesitancy were lack of information and fear of side effects. Vaccine acceptance was higher among males $(p<0.001)$, physicians $(p=0.017)$, participants with medical risk factors $(p=0.017)$, and participants vaccinated against influenza $(p<0.001)$. After control for other factors, male (OR, 1.94; CI 1.42-2.66), married participant (OR, 1.89; CI 1.22-2.92), living in a less crowded accommodation (OR, 1.33; CI 1.11-1.59), and who got influenza vaccine (OR, 1.64; CI 1.13-2.37) tended to accept the COVID-19 vaccine more likely.

Conclusion:

Rates of vaccine hesitancy among health care workers were high. The current pandemic offers an opportunity to establish better vaccine confidence towards the COVID-19 vaccine and vaccines in general.

Keywords: COVID-19, SARS-CoV-2, Vaccine, Vaccine hesitancy, Health care workers, Middle East.

\begin{tabular}{|l|l|l|l}
\hline Article History & Received: January 2, 2021 & Revised: May 16, 2021 & Accepted: May 25, 2021
\end{tabular}

\section{INTRODUCTION}

At the end of 2019, in Wuhan, a group of pneumonia cases of unknown etiology spread rapidly to cause an epidemic in China, which subsequently was disseminated worldwide. The

\footnotetext{
Address correspondence to this author at Department of Pediatrics, Faculty of Medicine, Cairo University, Giza, Egypt; Tel: +974 50056807;
}

E-mail:ahmadaon@hotmail.com disease was labeled coronavirus disease 2019 or COVID-19 [1]. The disease spread globally, primarily through person-toperson respiratory transmission [2]. By December 15th, 2020, there were 71 million confirmed cases of COVID19, including more than one and a half million deaths, according to the reports from the World Health Organization (WHO) [3]. Till the time of writing this manuscript, no cure is available for 
COVID-19. Of all the drugs being attempted, only dexamethasone has been beneficial in reducing mortality [4]. The best way to fight the COVID-19 pandemic is the development of a vaccine. Under normal circumstances, the vaccine development process involves the preclinical evaluation and three clinical phases: I, II, and III. This process is complicated and may need several years for completion [5]. Worldwide, the COVID-19 pandemic overwhelmed the health care systems, created an economic crisis, and disturbed societies [6]. For these reasons, the development of a COVID-19 vaccine has speeded up at an extraordinary rate. Various clinical vaccine trials are in progress. It is expected that a safe and effective vaccine will be available by $2020-2022$ [7]. At the time of preparation of this manuscript, results of phase III efficacy trials have been reported for some COVID-19 vaccines [8 - 10].

The fast-tracked development of COVID-19 vaccines has led to many concerns about safety and efficacy. This led to a rising vaccine hesitancy which can make widespread vaccine application more challenging. In a global survey of the potential acceptance of the COVID-19 vaccine, $28.5 \%$ of participants were hesitant to take the vaccine if it becomes available (range from $11.4 \%$ to $45.2 \%$ according to the country) [11]. Another survey among US adults found a hesitancy rate of $42.4 \%$ towards any future COVID-19 vaccine [12].

Vaccine hesitancy is defined as any delay in acceptance or refusal of a vaccine despite the availability of the vaccination services [13]. Vaccine hesitancy is one of the top 10 global health threats, according to the WHO. The WHO identified complacency, inconvenience, and lack of confidence as the major causes underlying hesitancy [14].

In addition to the higher risk of infection due to closer contact with patients, health care workers (HCWs) are exposed to enormous psychological stress as they can transmit the infection to their patients, their families, and the community in general $[15,16]$. HCWs are expected to have a more positive attitude towards any future COVID-19 vaccine and to have a prompt response to any public health crisis. However, rates of acceptance of any future COVID-19 vaccine among HCWs are still not clear. One study found that $48 \%$ of $\mathrm{HCWs}$ are hesitant to take the COVID-19 vaccine when it becomes available [17]. This contradiction needs further assessment because HCWs' acceptance of the COVID-19 vaccine is crucial. HCWs will be prioritized to get the vaccine once it becomes available [18]. Furthermore, HCWs are the most reliable advisors of vaccination decisions as they provide the community with trusted information about vaccines. However, an empty hand has nothing to give. If $\mathrm{HCW}$ are not accepting the vaccine, they will not recommend it to the community.

HCWs' concerns about the vaccine must be addressed and any efforts to improve COVID-19 vaccine uptake should identify reasons for vaccine hesitancy and use that information to establish trust in any future COVID-19 vaccine. In this study, we explored attitudes towards COVID-19 vaccine among HCWs in the Middle East region and we tried to find the reasons that lie beneath any vaccine hesitancy.

\section{MATERIALS AND METHODS}

\subsection{Research Design and Settings}

Our study was a cross-sectional study aiming at exploring HCWs' attitudes towards any future COVID-19 vaccine and to identify the reasons behind any vaccine hesitancy. An anonymous online self-reported questionnaire, hosted via Google forms, was sent out to a convenience sample of HCWs living and working in the Middle East region. The questionnaire was open from December $12^{\text {th }}$ to December $19^{\text {th }}$, 2020.

\subsection{Data Collection Tool (the Questionnaire)}

The questionnaire began with consent to participate. Then, a section containing questions covering the following items: demographic data, education level, presence of any of the following risk factors for severe COVID-19 infection (e.g., age $\geq 65$ years and associated medical conditions such as diabetes mellitus, hypertension, kidney disease, lung disease, liver disease, cardiac disease, immunosuppression, active cancer, and smoking) [19, 20], occurrence of previous COVID-19 infection, influenza vaccination history, and household crowding (crowded house was defined as more than one person per room and severely crowded was defined as more than 1.5 persons per room) [21]. The last section contained a direct question about COVID-19 vaccine and whether the participants would accept the vaccine when it becomes available in their country. Responses were ("yes", "no", or "not sure"). If participants chose "yes", they were asked two further questions. The first question was whether the participants were still willing to get the vaccine if it is not obligatory by their employer. The second question was about the reasons that make the participants willing to get the vaccine. If the participants chose "no" or "not sure", they were asked two further questions. The first question was about the reasons for hesitancy to get the vaccine. The second question was about the reasons that can convince the participants to get the vaccine.

\subsection{Statistical Analysis}

We aimed for a minimum sample size of 597 participants to ensure a confidence level of $95 \%$ and a margin of error within $\pm 4 \%$ of the surveyed values. Validated data were tabulated, entered, and analyzed using Statistical Package for the Social Sciences (SPSS) version 22.0 (SPSS Inc., Chicago, IL, USA). Chi-square $\chi 2$ test was used to test the impact of different variables on the participants' attitudes towards the COVID-19 vaccine. A $P$-value $<0.05$ was considered to be statistically significant. Logistic regression analysis was used to test the impact of different variables on participants' attitudes towards vaccination. The association between determinants and outcome are presented as odds ratio (OR) and 95\% confidence interval (CI) after controlling for other variables.

\subsection{Ethical Consideration}

The information was gathered from participants after obtaining their informed consent about the survey. No 
personally identifiable information was collected. Data confidentiality was assured. The research was approved by the Jahra hospital research committee (J1-12122020).

\section{RESULTS}

\subsection{Participants' Characteristics}

We received a total of 877 online responses to the survey. After the removal of duplicate responses (13/877), we had a total of 864 validated responses from HCWs living and working in the Middle East region (Kuwait, Egypt, Saudi Arabia, Qatar, Jordan, Iraq, Bahrain, Lebanon, Syria, and other countries). Their characteristics are summarized in Table $\mathbf{1}$. The study population included physicians $(42.2 \%)$, nurses (45.3\%), and other allied professionals (12.5\%). Females represented $61 \%$ of the study population. The majority of participants $(98.5 \%)$ were below 65 years old, married $(85.4 \%)$, and attained a university degree or a higher postgraduate degree $(97.7 \%)$. Comorbidities associated with COVID-19 severity were present in $28 \%$ of participants and approximately $71 \%$ of participants lived in crowded homes. Around $24 \%$ of HCWs in this study had a history of confirmed COVID-19 infection over the past year, as demonstrated in Table 1.

Approximately $32 \%$ of $\mathrm{HCWs}$ received the influenza vaccine in the last winter (2019), and $28 \%$ of them received it this winter (2020). The highest rate of influenza vaccination was among physicians (47\% in 2019 and $45 \%$ in 2020). It was noticed that the numbers of HCWs who received the influenza vaccine this year are less compared to the previous year as shown in Fig. (1).

Table 1. Description of participants' characteristics, overall and by occupation.

\begin{tabular}{|c|c|c|c|c|c|c|c|c|}
\hline & \multicolumn{2}{|c|}{$\begin{array}{c}\text { Total } \\
(\mathrm{n}=\mathbf{8 6 4})\end{array}$} & \multicolumn{2}{|c|}{$\begin{array}{l}\text { Physicians } \\
(\mathbf{n}=365)\end{array}$} & \multicolumn{2}{|c|}{$\begin{array}{l}\text { Nurses } \\
(\mathrm{n}=391)\end{array}$} & \multicolumn{2}{|c|}{$\begin{array}{l}\text { Allied Professions } \\
\quad(n=108)\end{array}$} \\
\hline & $\mathrm{n}$ & $\%$ & $\mathrm{n}$ & $\%$ & $\mathrm{n}$ & $\%$ & $\mathrm{n}$ & $\%$ \\
\hline \multicolumn{9}{|l|}{ Age Groups } \\
\hline 18-24years & 27 & 3.1 & 12 & 3.3 & 1 & 0.3 & 14 & 13 \\
\hline 25-34years & 237 & 27.4 & 86 & 23.6 & 111 & 28.4 & 40 & 37 \\
\hline 35-44years & 401 & 46.6 & 174 & 47.7 & 195 & 49.9 & 32 & 29.6 \\
\hline 45-54years & 145 & 16.8 & 59 & 16.2 & 75 & 19.2 & 11 & 10.2 \\
\hline 55-64years & 40 & 4.6 & 21 & 5.8 & 9 & 2.3 & 10 & 9.3 \\
\hline$\geq 65$ years & 14 & 1.5 & 13 & 3.6 & 0 & 0 & 1 & 0.9 \\
\hline \multicolumn{9}{|l|}{ Gender } \\
\hline Male & 338 & 39.1 & 239 & 65.5 & 50 & 12.8 & 49 & 45.4 \\
\hline Female & 526 & 60.9 & 126 & 34.5 & 341 & 87.2 & 59 & 54.6 \\
\hline \multicolumn{9}{|l|}{ Marital Status } \\
\hline Married & 738 & 85.4 & 299 & 81.9 & 366 & 93.6 & 73 & 67.6 \\
\hline Single $^{a}$ & 126 & 14.6 & 66 & 18.1 & 25 & 6.4 & 35 & 32.4 \\
\hline \multicolumn{9}{|c|}{ Educational Attainment } \\
\hline Higher postgraduate degree & 324 & 37.5 & 78 & 21.4 & 358 & 91.6 & 84 & 77.8 \\
\hline University/college degree & 520 & 60.2 & 287 & 78.6 & 16 & 4.1 & 21 & 19.4 \\
\hline High school & 20 & 2.3 & 0 & 0 & 17 & 4.3 & 3 & 2.8 \\
\hline \multicolumn{9}{|c|}{ Household Crowding $^{\mathrm{b}}$} \\
\hline Not crowded & 251 & 29.1 & 117 & 32.1 & 117 & 29.9 & 17 & 15.7 \\
\hline Crowded & 267 & 30.9 & 137 & 37.5 & 81 & 20.7 & 49 & 45.4 \\
\hline Severely crowded & 346 & 40 & 111 & 30.4 & 193 & 49.4 & 42 & 38.9 \\
\hline \multicolumn{9}{|l|}{ Comorbidities $^{c}$} \\
\hline None & 622 & 72 & 241 & 66 & 305 & 78 & 76 & 70.4 \\
\hline Yes & 242 & 28 & 124 & 34 & 86 & 22 & 32 & 29.6 \\
\hline \multicolumn{9}{|c|}{ COVID-19 Severity Risk Factors ${ }^{\mathrm{c}}$} \\
\hline None & 619 & 71.6 & 238 & 65.2 & 305 & 78 & 76 & 70.4 \\
\hline Age or comorbidities & 234 & 27.1 & 117 & 32.1 & 86 & 22 & 31 & 28.7 \\
\hline Age and comorbidities & 11 & 1.3 & 10 & 2.7 & 0 & 0 & 1 & 0.9 \\
\hline \multicolumn{9}{|c|}{ History of Confirmed COVID-19 Infection } \\
\hline No & 659 & 76.3 & 306 & 83.8 & 266 & 68 & 87 & 80.6 \\
\hline Yes & 205 & 23.7 & 59 & 16.2 & 125 & 32 & 21 & 19.4 \\
\hline
\end{tabular}

${ }^{a}$ Includes widowed and divorced.

${ }^{\mathrm{b}}[21]$

${ }^{\mathrm{c}}[19,20]$. 


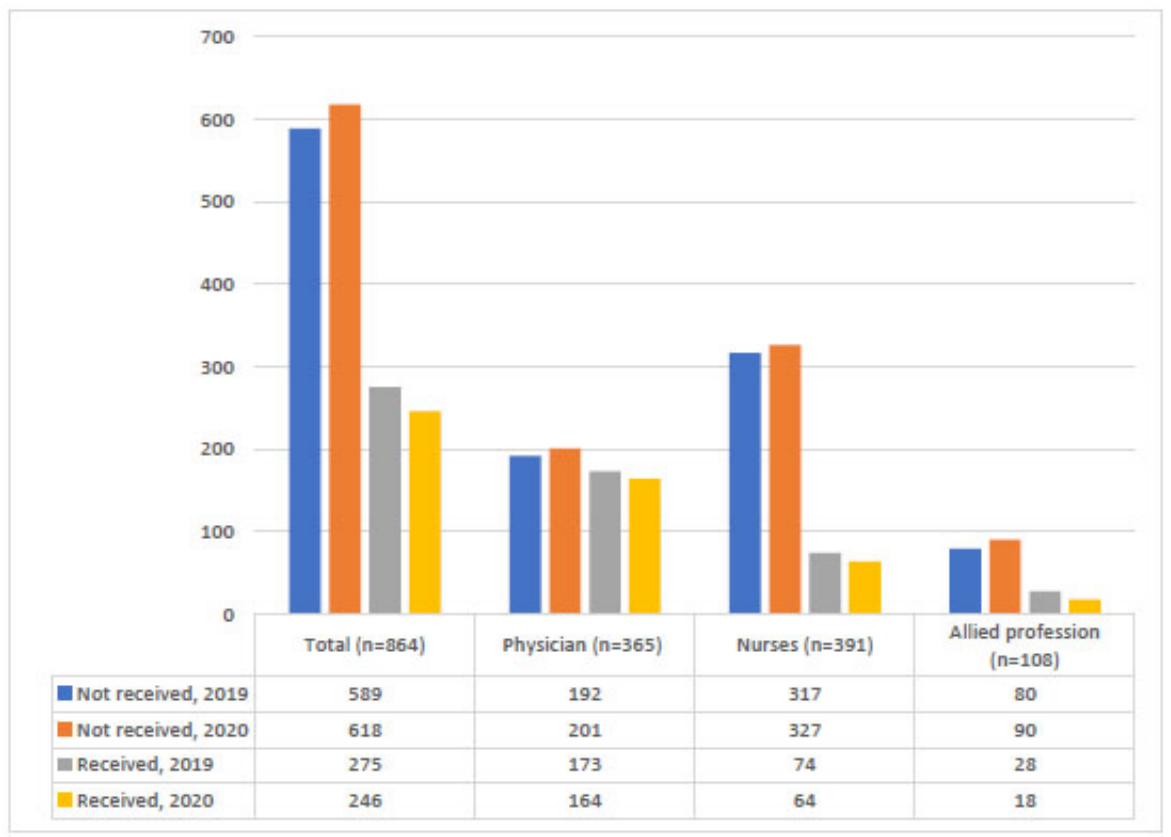

Fig. (1). Influenza vaccine status among health care workers 2019-2020, overall and by occupation.

\subsection{Vaccine Hesitancy and Associated Factors}

Overall, $40.7 \%$ of HCWs intended to get COVID-19 vaccine when it becomes available, $40.1 \%$ were not sure whether they would be vaccinated, and $19.2 \%$ refused the vaccine. The total rate of vaccine hesitancy was $59.3 \%$ and physicians tended to have a lower rate of vaccine hesitancy (53.7\%) when compared to nurses and allied professionals (63.2\% and 63.9\%, respectively), as shown in Fig. (2).

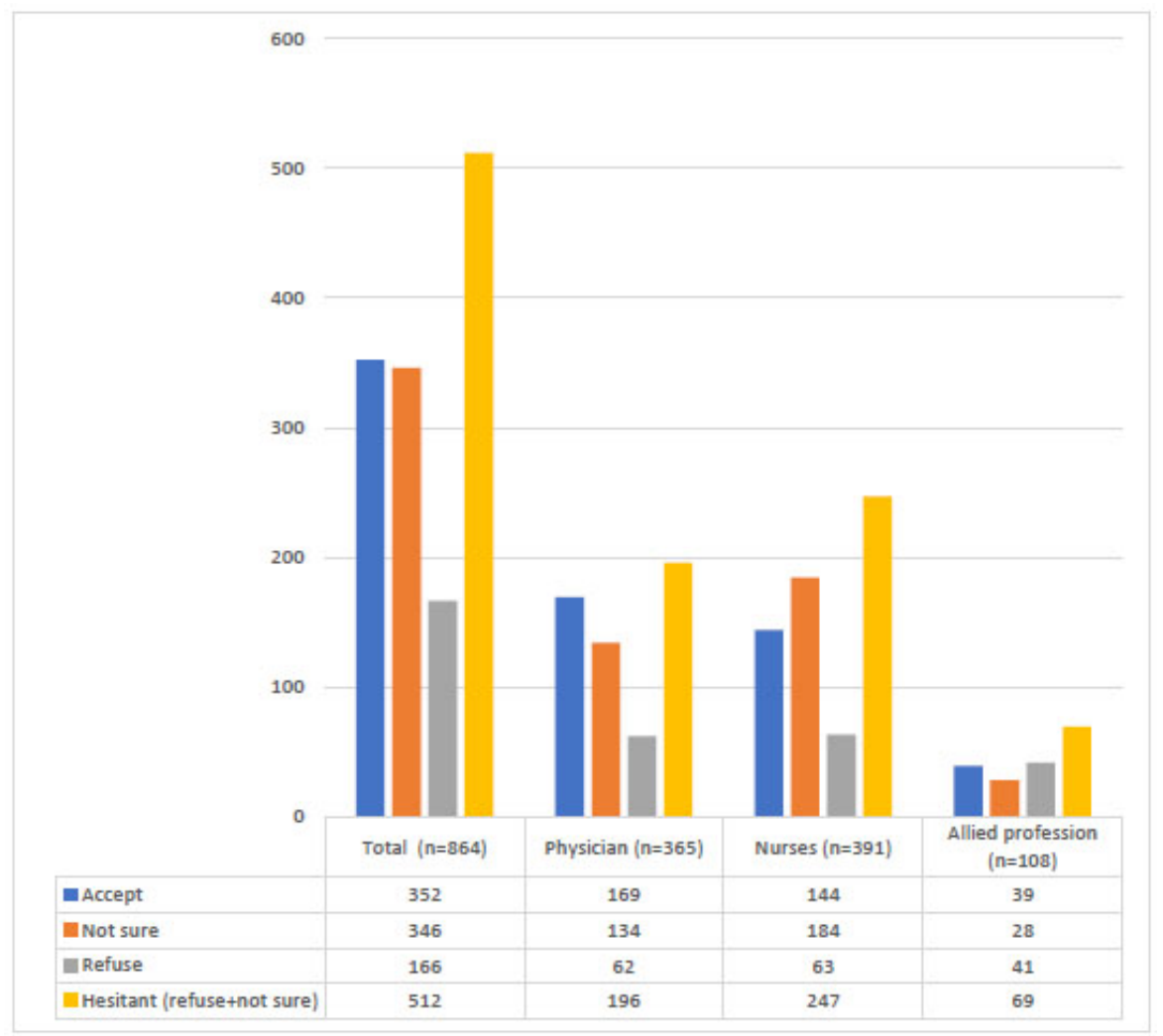

Fig. (2). Likelihood of taking COVID-19 vaccine, overall and by occupation. 
Among vaccine hesitant HCWs, the commonest reasons for hesitancy were the lack of information about the vaccine (56.6\%), followed by the fear of side effects (37.1\%). A large proportion of HCWs stated that they would accept the vaccine if they had more information about the product $(37 \%)$ or if more studies confirmed the efficacy and safety of the immunization (48\%), as shown in Table 2.

Among HCWs who accepted the vaccine, most of them reported taking the vaccine to protect themselves or protect others against the virus ( $71 \%$ and $17.6 \%$, respectively), while the remaining $11.4 \%$ of $\mathrm{HCWs}$ accepted the vaccine to avoid any travel or work restrictions. A large proportion of them (72.4\%) were still willing to take the vaccine even if it was not obligatory by their employers or health authorities.

Table 3 summarizes the effect of different variables on HCWs' attitudes towards COVID-19 vaccine. The gender difference was significant; male HCWs tended to accept the vaccine more than female HCWs $(p<0.001)$. There was no significant difference in attitudes among different age groups $(p=0.627)$ or marital status groups $(p=0.43)$. HCWs with higher educational attainment and physicians had significantly higher levels of vaccine acceptance $(p=0.011$ and 0.017 , respectively). We also found that acceptance rates among younger HCWs ( $<65$ years) without medical risk factors to be $38 \%$, rising to $47 \%$ in younger HCWs with medical risk factors, and reaching up to $64 \%$ in older HCWs ( $\geq 65$ years) with medical risk factor $(p=0.017)$. Strikingly, we found that HCWs who lived in crowded houses show more negative attitudes towards COVID-19 vaccination compared to HCWs living in non-crowded houses $(p=0.006)$. Unsurprisingly, HCWs who received the influenza vaccine tended to accept COVID-19 vaccine more than those who did not $(p<0.001)$.

Table 2. Concerns of hesitant health care workers about COVID-19 vaccine, overall and by occupation.

\begin{tabular}{|c|c|c|c|c|c|c|c|c|}
\hline & \multicolumn{2}{|c|}{$\begin{array}{c}\text { Physicians } \\
(n=196)\end{array}$} & \multicolumn{2}{|c|}{$\begin{array}{l}\text { Nurses } \\
(n=247)\end{array}$} & \multicolumn{2}{|c|}{$\begin{array}{c}\text { Allied Professions } \\
(n=69)\end{array}$} & \multicolumn{2}{|c|}{$\begin{array}{c}\text { Total } \\
(n=512)\end{array}$} \\
\hline & $\mathrm{n}$ & $\%$ & $\mathrm{n}$ & $\%$ & $\mathrm{n}$ & $\%$ & $\mathrm{n}$ & $\%$ \\
\hline \multicolumn{9}{|l|}{ Concerns of hesitant health care workers } \\
\hline Lack of information & 110 & 56.1 & 140 & 56.7 & 40 & 58 & 290 & 56.6 \\
\hline Possible side effects & 74 & 37.8 & 94 & 38.1 & 22 & 31.9 & 190 & 37.1 \\
\hline Others $^{a}$ & 12 & 6.1 & 13 & 5.3 & 7 & 10.1 & 32 & 6.3 \\
\hline \multicolumn{9}{|l|}{ What can convince hesitantHCWs to accept the vaccine } \\
\hline More information about the vaccine & 54 & 27.6 & 117 & 47.4 & 19 & 27.5 & 190 & 37.1 \\
\hline More studies on vaccine safety and efficacy & 114 & 58.2 & 105 & 42.5 & 29 & 42 & 248 & 48.4 \\
\hline Official health authority's recommendation & 9 & 4.6 & 13 & 5.3 & 9 & 13 & 31 & 6.1 \\
\hline Will never accept & 19 & 9.6 & 12 & 4.8 & 12 & 17.5 & 43 & 8.4 \\
\hline
\end{tabular}

ancludes worries about vaccine effectiveness and cost or persons who are against vaccines in general.

Table 3. Intent to get the vaccine among health care workers, by participants characteristics.

\begin{tabular}{|c|c|c|c|}
\hline Characteristics & Vaccine hesitant $(\mathrm{n}=512), \mathrm{n}(\%)$ & Accept vaccine $(n=352), n(\%)$ & $P$-value \\
\hline \multicolumn{3}{|l|}{ Age Group } & 0.627 \\
\hline 18-24years & $17(3.3)$ & $10(2.8)$ & \\
\hline 25-44years & $380(74.2)$ & $258(73.3)$ & \\
\hline 45-64years & $109(21.3)$ & $76(21.6)$ & \\
\hline$\geq 65$ years & $6(1.2)$ & $8(2.3)$ & \\
\hline \multicolumn{3}{|l|}{ Gender } & $<0.001$ \\
\hline Male & $161(31.4)$ & $177(50.3)$ & \\
\hline Female & $351(68.6)$ & $175(49.7)$ & \\
\hline \multicolumn{3}{|l|}{ Marital Status } & 0.43 \\
\hline Single $^{a}$ & $85(16.6)$ & $41(11.6)$ & \\
\hline Married & $427(83.4)$ & $311(88.4)$ & \\
\hline \multicolumn{3}{|c|}{ Educational Attainment } & 0.011 \\
\hline High school & $13(2.5)$ & $7(2)$ & \\
\hline University/college degree & $328(64.1)$ & $192(54.5)$ & \\
\hline Higher postgraduate degree & $171(33.4)$ & $153(43.5)$ & \\
\hline \multicolumn{3}{|l|}{ Occupation } & 0.017 \\
\hline Physicians & $196(38.3)$ & $169(48)$ & \\
\hline Nurses & $247(48.2)$ & $144(40.9)$ & \\
\hline Allied professionals & $69(13.5)$ & $39(11.1)$ & \\
\hline
\end{tabular}




\begin{tabular}{|c|c|c|c|}
\hline Characteristics & Vaccine hesitant $(\mathrm{n}=512), \mathrm{n}(\%)$ & Accept vaccine $(n=352), n(\%)$ & $P$-value \\
\hline \multicolumn{3}{|c|}{ Household Crowding $^{\text {b }}$} & 0.006 \\
\hline Not crowded & $128(25)$ & $123(34.9)$ & \\
\hline Crowded & $164(32)$ & $103(29.3)$ & \\
\hline Severely crowded & $220(43)$ & $126(35.8)$ & \\
\hline \multicolumn{3}{|c|}{ COVID-19 Severity Risk Factors ${ }^{\mathrm{c}}$} & 0.017 \\
\hline None & $384(75)$ & $235(66.7)$ & \\
\hline Age or comorbidities & $124(24.2)$ & $110(31.3)$ & \\
\hline Age and comorbidities & $4(0.8)$ & $7(2)$ & \\
\hline \multicolumn{3}{|c|}{ Influenza Vaccination, 2019} & $<0.001$ \\
\hline No & $376(73.4)$ & $213(60.5)$ & \\
\hline Yes & $136(26.6)$ & $139(39.5)$ & \\
\hline \multicolumn{3}{|c|}{ Influenza Vaccination, 2020} & $<0.001$ \\
\hline No & $394(77)$ & $224(63.6)$ & \\
\hline Yes & $118(23)$ & $128(36.4)$ & \\
\hline
\end{tabular}

${ }^{\mathrm{a}}$ Includes widowed and divorced.

${ }^{\mathrm{b}}[21]$

c $[19,20]$.

\subsection{Determinants of Vaccine Acceptance}

Logistic regression analysis was used to analyze determinants associated with vaccine acceptance among HCWs after control for other confounders. We found that male (OR, 1.94; 95\% CI 1.42-2.66; $p<0.001$ ), married HCWs (OR, 1.89; $95 \%$ CI 1.22-2.92; $p=0.004)$, who live in less crowded houses (OR, 1.33; 95\% CI 1.11-1.59; $p=0.002)$ and who received the influenza vaccine this year (OR, 1.64; 95\% CI 1.13-2.37; $p=0.009$ ) tended to accept the COVID-19 vaccine more than other HCWs after control for other factors like age, educational level, occupation, and the presence of risk factors for COVID-19 severity.

\section{DISCUSSION}

COVID-19 vaccine is expected to provide immunity to the immunized resulting in lower infection rates in the community as well as reduced COVID-19 hospitalizations. COVID-19 vaccine coverage necessary to achieve population immunity was estimated to be at least $70 \%$ of the community [12]. However, some groups of the population will not be eligible for COVID-19 vaccination, such as children and adolescents, persons within 90 days of COVID-19 infection, pregnant women, and those with a history of severe allergic disease [22]. Besides, any COVID-19 vaccine will not be $100 \%$ effective. Current vaccines confer $62-95 \%$ protection rate at best $[8,10]$. Any further vaccine hesitancy will additionally deter the achievement of vaccination goals. It is obvious that the extent of benefit is greater with increased coronavirus vaccination coverage; nevertheless, vaccine hesitancy is rising.

HCWs are expected to be a good example in their health behaviors. HCWs are an open-minded and well-educated population supposed to have the highest vaccine acceptance rates. Contrary to these expectations, we found a high rate of vaccine hesitancy among HCWs, with nurses and allied professionals showing more vaccine hesitancy when compared to physicians. Our results are in accordance with the previous reports demonstrating high rates of COVID-19 vaccine hesitancy among HCWs and the general population [11, 12, 17].
As far as we know, no research has investigated rates of COVID-19 vaccine hesitancy among HCWs compared to the general population. A recent study comparing healthcare university students versus non-healthcare university students found no significant differences in vaccine hesitancy rates. These findings are striking and need special attention [23].

The principle of nonmaleficence oblige HCWs not to harm their patients. Some will argue that vaccination against COVID-19 should be mandatory for all HCWs because caring for patients without vaccination is maleficent and can enhance nosocomial transmission of the virus. However, reports from the USA suggested that the risk of nosocomial COVID-19 transmission was exceptionally low, and strict infection control measures made nosocomial COVID-19 a rare event [24]. We found that the majority of HCWs who accepted the vaccine were willing to get vaccinated irrespective of any obligations from their employers or health authorities. Similar findings were reached by other studies suggesting that encouraging voluntary vaccine acceptance is a better choice [11].

The concerns of hesitant HCWs were related mainly to insufficient knowledge about the vaccine and the potential side effects. Other studies reported that inadequate knowledge and concerns about the safety profile were the commonest reasons for COVID-19 vaccination hesitancy among HCWs [17, 25]. These misconceptions are usually empowered by conspiracy theories and fake news on social media platforms [26]. This may explain the higher rates of acceptance among physicians who usually have better knowledge about vaccines when compared to other HCWs. HCWs are struggling to keep pace with the growing body of evidence and the hundreds of papers published daily on COVID-19. Any information campaign directed to HCWs must be concise, accurate, evidence-based, and aiming to raise HCWs' knowledge regarding vaccination.

Older age and certain comorbidities are associated with COVID-19 severity $[19,20]$. In our study, a higher number of risk factors for severity was associated with a more likelihood of accepting the vaccine. This is expected as they are the most vulnerable groups and taking the vaccine will be in their best interest. 
Some of the HCWs who accepted vaccination in our study reported that they will get vaccinated to avoid any travel or work restrictions. Exempting vaccinated persons from travel restrictions or any other social distancing activities can be used as an encouragement to uptake the vaccine.

Among all the tested variables, male gender, married marital status, less household crowding, and influenza vaccination acceptance were associated with higher rates of COVID-19 vaccine acceptance.

Higher rates of COVID-19 vaccine acceptance among male HCWs may be explained by the tendency towards a risktaking behavior because of the shortage of information about the new vaccine [27].

The main concern of HCWs is usually transmitting the infection to others, especially to their families, as demonstrated in previous studies [15]. This can explain why married HCWs are more likely to accept COVID-19 vaccine.

HCWs living in crowded houses had a negative attitude towards COVID-19 vaccine. We speculate that this might be due to a lower socioeconomic status. Previous studies showed that people with lower income and lower educational attainment were less likely to accept the vaccine [11, 12].

Even before the COVID-19 pandemic, vaccine hesitancy among HCWs already existed towards other vaccines such as the influenza ones, despite the strong recommendation of getting that immunization for all HCWs [28, 29]. In spite of the ongoing COVID-19 pandemic and the increased awareness of respiratory viral diseases, rates of influenza vaccination among surveyed HCWS this year were lower compared to the previous year. In our study, a large proportion of HCWs did not receive the influenza vaccine and was hesitant to receive any future COVID-19 vaccine. The negative attitudes towards both vaccines suggest that hesitancy is not only due to some concerns about safety or efficacy but could be due to false ideas related to the vaccination process in general. In line with our findings, other studies demonstrated that influenza vaccination hesitancy is a risk factor for COVID-19 vaccine hesitancy $[12,17]$

A limitation of the present study is that our sample is not fully representative of all the Middle East HCWs. However, our results can help in the understanding of the magnitude of vaccine hesitancy and its underlying causes. It is important to note that vaccination choices are complex and can change over time. HCWs accepting the vaccine now can refuse it in the future, and vice versa. One strength in our study was that we conveyed our survey at a time when a vaccine was available and approved for use, contrary to the other surveys done before the time of vaccine availability. The identification of vaccine hesitancy risk factors can help health authorities to efficiently target messages about COVID-19 vaccination to the proper audience.

\section{RECOMMENDATION}

The findings of this study should be taken as a warning of the rising COVID-19 vaccine hesitancy among HCWs. Governments should listen to HCWs and address their concerns. Health authorities must tailor strategies to counteract fake information and improve confidence in vaccination campaigns among HCWs. Building confidence in COVID-19 vaccine by campaigns that assure effectiveness and safety is crucial. These campaigns should target both HCWs and the public to ensure wide acceptance of the vaccine. The content of these campaigns should be tailored to fit each group, with a more technical and professional message directed to HCWs. This knowledge will encourage HCWs to deliver a positive message to the general public. The vaccination program must be supported by civil and religious organizations through accurate information spread in the community. Introducing curricula about vaccination for all health-care students can help improving vaccine acceptance among future HCWs. Nevertheless, it is of paramount importance to understand that there is no universal strategy to resolve vaccine hesitancy and that joint efforts are needed. Any intervention should be tailored to suit local cultural concepts and should be maintained over time.

\section{CONCLUSION}

In conclusion, rates of vaccine hesitancy among HCWs were elevated.We think that our results merit attention in light of the ongoing vaccination campaigns. We expected that HCWs would have higher levels of vaccine acceptance. Further studies are needed for a better understanding of the nature of the hesitancy among HCWs. The current pandemic offers the best chance to establish better vaccine confidence towards COVID-19 vaccine and vaccines in general. Any action or policy must be transparent and accurate to foster this confidence.

\section{ETHICS APPROVAL AND CONSENT TO PARTICIPATE}

The research was approved by the research committee of Jahra hospital, Kuwait (J1-12122020).

\section{HUMAN AND ANIMAL RIGHTS}

No animals were used in this research. All human research procedures followed were in accordance with the ethical standards of the committee responsible for human experimentation (institutional and national) and with the Helsinki Declaration of 1975, as revised in 2013.

\section{CONSENT FOR PUBLICATION}

Informed consent was obtained from the participants prior to data collection.

\section{FUNDING}

None.

\section{AVAILABILITY OF DATA AND MATERIALS}

Data will be available upon reasonable request from the corresponding author and after approval from all authors.

\section{CONFLICT OF INTEREST}

The authors declare no conflicts of interest, financial or otherwise. 


\section{ACKNOWLEDGEMENTS}

We would like to acknowledge all health care workers who participated in the survey.

\section{REFERENCES}

[1] World Health Organization (WHO). WHO Director-General's remarks at the media briefing on 2019-nCoV on. 2020. Available from: https://www.who.int/director-general/speeches/detail/who-director-gen eral-s-remarks-at-the-media-briefing-on-2019-ncov-on-11february-2020

[2] Meyerowitz EA, Richterman A, Gandhi RT, Sax PE. Transmission of SARS-CoV-2: A review of viral, host, and environmental factors. Ann Intern Med 2020; 17: M20-M5008

[http://dx.doi.org/10.7326/M20-5008] [PMID: 32941052]

[3] World Health Organization(WHO). WHO Coronavirus Disease (COVID-19) Dashboard.. Available from: https://covid19.who.int/

[4] Horby P, Lim WS, Emberson JR, et al. A. On behalf of RECOVERY Collaborative Group. Dexamethasone in hospitalized patients with COVID-19 - preliminary report. N Engl J Med 2020; 17

[5] Mitchell VS, Philipose NM, Sanford JP, Eds. Philipose VS, Sanford NM, Sanford JP, Eds. Stages of Vaccine Development In: The Children's Vaccine Initiative: Achieving the Vision; Mitchell,. National Academies Press (US):Washington (DC) 1993; p. 109.

[6] Nicola M, Alsafi Z, Sohrabi C, et al. The socio-economic implications of the coronavirus pandemic (COVID-19): A review. Int J Surg 2020; 78: $185-93$

[http://dx.doi.org/10.1016/j.ijsu.2020.04.018] [PMID: 32305533]

[7] Callaway E. The race for coronavirus vaccines: A graphical guide. Nature 2020; 580(7805): 576-7

[http://dx.doi.org/10.1038/d41586-020-01221-y] [PMID: 32346146]

[8] Polack FP, Thomas SJ, Kitchin N, et al. Jr.; Hammitt, L.L.; Türeci, Ö.; Nell, H., Schaefer, A., Ünal, S. Safety and efficacy of the BNT162b2 mRNA Covid-19 Vaccine. N Engl J Med 2020.

[http://dx.doi.org/10.1056/NEJMoa2034577]

[9] Moderna. Moderna announces primary efficacy analysis in phase 3 cove study for its COVID-19 vaccine candidate and filing today with US FDA for emergency use authorization Available from: https://investors.modernatx.com/news-releases/news-release-details/m oderna-announces-primary-efficacy-analysis-phase-3-cove-study/

[10] Voysey M, Clemens SAC, Madhi SA, et al. Group, Oxford COVID Vaccine Trial. Safety and efficacy of the ChAdOx $1 \mathrm{nCoV}-19$ vaccine (AZD1222) against SARS-CoV-2: An interim analysis of four randomized controlled trials in Brazil, South Africa, and the UK. Lancet 2020; S0140-6736(20): 32661-1.

[11] Lazarus JV, Ratzan SC, Palayew A, et al. A global survey of potential acceptance of a COVID-19 vaccine. Nat Med 2020 [PMID: 33082575]

[12] Fisher KA, Bloomstone SJ, Walder J, Crawford S, Fouayzi H, Mazor KM. Attitudes toward a potential SARS-CoV-2 vaccine : A survey of U.S. adults. Ann Intern Med 2020; 173(12): 964-73.

[http://dx.doi.org/10.7326/M20-3569] [PMID: 32886525]

[13] MacDonald NE. SAGE Working group on vaccine hesitancy. Vaccine hesitancy: Definition, scope and determinants. Vaccine 2015; 33(34): 4161-4.

[http://dx.doi.org/10.1016/j.vaccine.2015.04.036] [PMID: 25896383]

[14] World Health Organization (WHO). Ten threats to global health 2019. Available from: https://www.who.int/news-room/spotlight/ ten-threatsto-global-health-in-2019

[15] Aon MH, Al-Shammari OZ, Aljenfawi MK, Aoun AH. Psychological impact of the covid-19 pandemic on healthcare workers in Kuwait. American Journal of Research in Medical Sciences 2020; 5(1): 1-7.
[16] Aoun A, Abdel Rahman Y, Mostafa NM, Kassem I, Badrah M. Impact of the COVID-19 pandemic on health care workers' mental health: A cross-sectional study. Allied J Med Res 2020; 4(1): 57-62.

[17] Grech V, Gauci C, Agius S. Withdrawn: Vaccine hesitancy among Maltese Healthcare workers toward influenza and novel COVID-19 vaccination. Early Hum Dev 2020.105213 [published online ahead of print.].

[http://dx.doi.org/10.1016/j.earlhumdev.2020.105213]

[PMID: 33032879]

[18] Centers for Disease Control and Prevention (CDC); ACIP COVID-19 Vaccines Work Group. Phased Allocationof COVID-19 Vaccines Available from: https://www.cdc.gov/vaccines/acip/meetings/ downloads/slides-2020-12/COVID-02-Dooling.pdf

[19] CDC Morbidity and Mortality Weekly Report (MMWR). Severe Outcomes Among Patients with Coronavirus Disease 2019 (COVID-19) - United States. 2020. Available from: https://www. cdc.gov/mmwr/ volumes/69/wr/mm6912e2.htm

[20] Williamson EJ, Walker AJ, Bhaskaran K, et al. Factors associated with COVID-19-related death using OpenSAFELY. Nature 2020; 584(7821): 430-6.

[http://dx.doi.org/10.1038/s41586-020-2521-4] [PMID: 32640463]

[21] United States census bureau historical census of housing tables: Crowding 2020. Available from: https://www.census.gov/data/ tables/time-series/dec/coh-crowding.html

[22] Centers for Disease Control and Prevention (CDC). Interim clinical considerations for use of mRNA COVID-19 vaccines currently authorized in the United States 2020. Available from: https://www.cdc.gov/ vaccines/covid-19/info-by-product/clinicalconsiderations.html

[23] Barello S, Nania T, Dellafiore F, Graffigna G, Caruso R. 'Vaccine hesitancy' among university students in Italy during the COVID-19 pandemic. Eur J Epidemiol 2020; 35(8): 781-3.

[http://dx.doi.org/10.1007/s10654-020-00670-z] [PMID: 32761440]

[24] Rhee C, Baker M, Vaidya V, et al. CDC prevention epicenters program. Incidence of Nosocomial COVID-19 in Patients Hospitalized at a Large US Academic Medical Center. JAMA Netw Open 2020; 3(9)e2020498

[http://dx.doi.org/10.1001/jamanetworkopen.2020.20498] [PMID: 32902653]

[25] Grech V, Bonnici J, Zammit D. WITHDRAWN: Vaccine hesitancy in Maltese family physicians and their trainees vis-à-vis influenza and novel COVID-19 vaccination. Early Hum Dev 2020.105259 [http://dx.doi.org/10.1016/j.earlhumdev.2020.105259]

[PMID: $33213968]$

[26] Puri N, Coomes EA, Haghbayan H, Gunaratne K. Social media and vaccine hesitancy: New updates for the era of COVID-19 and globalized infectious diseases. Hum Vaccin Immunother 2020; 16(11): 2586-93.

[http://dx.doi.org/10.1080/21645515.2020.1780846]

[PMID: 32693678]

[27] Turner C, McClure R. Age and gender differences in risk-taking behaviour as an explanation for high incidence of motor vehicle crashes as a driver in young males. Inj Control Saf Promot 2003; 10(3): 123-30.

[http://dx.doi.org/10.1076/icsp.10.3.123.14560] [PMID: 12861910]

[28] Wilson R, Zaytseva A, Bocquier A, et al. Vaccine hesitancy and selfvaccination behaviors among nurses in southeastern France. Vaccine 2020; 38(5): 1144-51.

[http://dx.doi.org/10.1016/j.vaccine.2019.11.018] [PMID: 31810781]

[29] Di Martino G, Di Giovanni P, Di Girolamo A, et al. Knowledge and Attitude towards Vaccination among Healthcare Workers: A Multicenter Cross-Sectional Study in a Southern Italian Region. Vaccines (Basel) 2020; 8(2): 248.

[http://dx.doi.org/10.3390/vaccines8020248] [PMID: 32456273] 